\title{
Survey of estrogenic activity in fish feed by YES assay
}

\author{
TAKERU MATSUMOTO, ${ }^{1}$ MAKITO KOBAYASHI, ${ }^{1 *}$ SHIN' ICHIRO KAWAI, ${ }^{2}$ TOSHIHISA MORIWAKI, ${ }^{3}$ AND \\ SHUGO WATABE ${ }^{1}$ \\ ${ }^{1}$ Department of Aquatic Bioscience, Graduate School of Agricultural and Life Sciences, University of Tokyo, \\ Bunkyo, Tokyo 113-8657 (amakito(omail.ecc.u-tokyo.ac.jp; Kobayashi), ${ }^{2}$ Department of Human Sciences, Kobe \\ College, Nishinomiya, Hyogo 662-8505, and ${ }^{3}$ Fish-Feed Section, Taiyo Shiryo Co., Ltd., Chita, Aichi 478-0046, \\ Japan
}

\section{KEY WORDS: fish feed, endocrine disruptors, phytoestrogen, YES assay}

\section{INTRODUCTION}

It is recently considered that some chemicals in the environment have the potential to adversely affect endocrine systems of human and wild animals.) These chemicals are called endocrine disruptors. Among these chemicals, some of them are reported to show estrogenic effects and are presumed to influence reproductive activity of human and wild animals including fish.

Plasma or hepatic vitellogenin (VTG) in male fish has been used as a specific biomarker for the detection of estrogen exposure. ${ }^{2,3)}$ VTG is a precursor protein of egg yolk in oviparous animals and produced in the liver under a regulation of estrogens in vertebrates. ${ }^{2)}$ Female fish show high plasma levels of VTG during ovarian development whereas plasma VTG levels in males are normally very low or non-detectable. ${ }^{4)}$ Since VTG production can be elicited in males by administration of exogenous estrogens, VTG in male fish is considered to be a good biomarker for estrogen exposure.

In our previous study (Matsumoto et al., submitted), a small amount of VTG was detected in the plasma of wild and cultured common carp of Lake Kasumigaura, Japan, and their testicular development seemed to be histologically normal. Although the cause of VTG production in these carps has not been identified, it is possible that testicular steroids and food, as well as endocrine disruptors, induced VTG synthesis in male fish.

In the present study, in order to examine possibility of VTG production by endogenous steroids and food, we have determined the estrogenic activity of animal steroids, phytosteroids, and fish feed by in vitro yeast estrogen-screen assay (YES assay). ${ }^{5}$ )

\section{MATERIALS AND METHODS}

Investigated animal steroids, related substances, and phytosteroids are shown in Table 1 . Standard chemicals were dissolved in dimethyl sulfoxide (DMSO) except $\beta$-sitosterol, which was dissolved in methanol.

Five commercial fish feeds (Table 2), four for cultured species, one for omamental fish, and one newly formulated fish feed without containing soybean (Kobayashi, in preparation), were investigated for their estrogenic activity by the YES assay. Five grams of fish feed was mixed with $50 \mathrm{ml}$ of methanol, sonicated for $10 \mathrm{~min}$, and centrifuged at $2000 \times g$ for $20 \mathrm{~min}$, and the supernatant was obtained as extract. The methanol extraction was conducted twice, and combined extracts were dried and dissolved in DMSO. DMSO or methanol solution of standard chemicals or fish feed extract dissolved in DMSO was served for the assay.

Estrogenic activity was measured by YES assay as described by Routledge et al. ${ }^{5}$ hER-yeast used in this assay has the DNA sequence of human estrogen receptor $\alpha$ in the chromosome and an expression plasmid carrying the estrogen responsive element and the reporter gene $l a c-Z$ which encodes $\beta$-galactosidase. When hER-yeast is cultured with the medium containing chlorophenol red- $\beta$-D-galactopyranoside for 3 days at $32{ }^{\circ} \mathrm{C}$ in the presence of estrogenic substance as a ligand, color development occurs in the medium in proportion to the amount of estrogenic substance. Absorbance at $540 \mathrm{~nm}$ of the medium was read to estimate estrogenic activity.

Data derived from each assay were fitted using a logistic dose-response equation to estimate the concentration-dependent effect and $50 \%$ efficiency of a ligand on transactivation. The relative potency ratio of standard chemicals and estradiol equivalent of fish feed extract were calculated from the concentration of $50 \%$ efficiency.

\section{RESULTS}

Among standard chemicals tested, estrogens, some androgens, and phytoestrogens showed estrogenic activity (Table 1). All the commercial fish feeds tested showed estrogenic activity, whereas no estrogenic 
activity was detected in the formulated fish feed (Table 2).

Table 1. Estrogenic activity of steroids and related substances

\begin{tabular}{ll}
\hline Standard chemicals & $\begin{array}{c}\text { Potency } \\
\text { ratio }\end{array}$ \\
\hline Animal Steroids & \\
\hline $17 \beta$-estradiol & 1 \\
ethynylestradiol & 0.74 \\
equilin & 0.084 \\
tamoxifen & $\mathrm{ND}$ \\
testosterone & $3.7 \times 10^{-6}$ \\
$5 \alpha$-dihydrotestosterone & $1.2 \times 10^{-4}$ \\
11 -ketotestosterone & $3.0 \times 10^{-6}$ \\
androstenedione & $\mathrm{ND}$ \\
11 -ketoandrostendione & $\mathrm{ND}$ \\
progesterone & $\mathrm{ND}$ \\
$17 \alpha, 20 \beta$-dihydroxy-4-pregnen-3-one & $\mathrm{ND}$ \\
$17 \alpha, 20 \beta, 21$-trihydroxy-4-pregnen-3-one & $\mathrm{ND}$ \\
ecdysone & $\mathrm{ND}$ \\
20 -hydroxyecdysone & $\mathrm{ND}$ \\
\hline Phytosteroids & \\
\hline ponasterone A & $\mathrm{ND}$ \\
$\beta$-sitosterol & $\mathrm{ND}$ \\
genistein & $8.6 \times 10^{-6}$ \\
daidzein & $\mathrm{ND}$ \\
biochanin A & $\mathrm{ND}$ \\
coumestrol & $5.4 \times 10^{-5}$ \\
formononetin & $4.6 \times 10^{-5}$ \\
+/-/-equol & $1.1 \times 10^{-4}$ \\
\hline
\end{tabular}

ND, not detectable in this system.

Table 2. Estrogenic activity of fish feed

\begin{tabular}{lc} 
Fish feed & $\begin{array}{c}\text { Estradiol } \\
\text { equivalent } \\
(\mathrm{ng} / \mathrm{g})\end{array}$ \\
\hline pellet for carp & 3.02 \\
pellet for trout & 1.06 \\
pellet for seabream & 2.49 \\
pellet for eel & 0.25 \\
pellet for ornamental carp & 1.29 \\
formulated fish feed & $\mathrm{ND}$ \\
\hline
\end{tabular}

\section{DISCUSSION}

In the present study, estrogenic activity was detected in some androgens, phytosteroids, and fish feeds although the activity was much lower than those of natural estrogens. Among androgens, testosterone, $5 \alpha$-dihydrotestosterone, and 11-ketotestosterone showed estrogenic activity, of which testosterone and 11-ketotestosterone are already known as major testicular androgens in fish. It is also known that small amount of estradiol is produced in the testis of fish. Therefore, it is possible that these estrogenic testicular steroids are involved in stimulating the synthesis of VTG.

Progestogens (progesterone, 17 $\alpha, 20 \beta$-dihydroxy-4-pregnene-3-one, and 17 $\alpha, 20 \beta, 21$-trihydroxy-4-pregnene-3-one), which are known to induce spermiation in male fish, showed no estrogenic activity, and it is unlikely that these steroids are involved in VTG production.
Ecdysones (ecdysone and 20-hydroxyecdysone), steroids produced in insects and crustaceans, which are often preyed by fish, did not show estrogenic activity.

Phytosteroids (genistein, coumestrol, formononetin, and equol) showed estrogenic activity. Since some species of fish are omnivorous or herbivorous, it is possible that fish ingest these phytosteroids in aquatic plants as food and that these phytosteroids stimulate VTG production in male fish.

All commercial fish feeds tested showed estrogenic activity. It is considered that estrogenic activity in the feed is attributed to phytosteroids in soybean since most of fish feed contain soybean as a source of protein and soybean is known to contain phytosteroids. $^{6,7)}$ In contrast, the soybean-free formulated feed did not show estrogenic activity.

It is highly probable that VTG detected in male culture carp was induced by fish feed. In fact, VTG production was induced in male goldfish when fish were fed commercial fish feed, whereas VTG levels were low when fish fed with the soybean-free formulated diet (Kobayashi, in preparation).

In summary, we surveyed estrogenic activity of steroids and fish feed, and observed that some androgens, phytosteroids, and fish feed had estrogenic activity. The results of the present study suggest that VTG production could be induced not only by endocrine disruptors but also by endogenous steroids and/or food containing phytosteroids. Furthermore, the soybean-free formulated fish diet would be useful for in vivo study of estrogenic endocrine disruptors.

\section{REFERENCES}

1. Kime DE. Endocrine disruption in fish. Kluwer Academic Publishers, Boston. 1998.

2. Hara A. Vitellogenin in male as a biomarker for estrogenic contamination of the aquatic environment. $J p m$. J. Environ. Toxicol. 1999; 2, 35-42.

3. Sumpter JP Jobling S. Vitellogenin as a biomarker for estrogenic contamination of the aquatic environment. Environ. Health Perspect. 1995; 103 (suppl. 7): 173-178.

4. Copeland PA, Sumpter JP, Walker TK, Croft M. Vitellogenin levels in male and female rainbow trout (Salmo gairdneri Richardson) at various stages of the reproductive cycle. Comp. Biochem. Physiol. 1986; 83B: 487-493.

5. Routledge EJ, Sumpter JP. Estrogenic activity of surfactants and some of their degradation products assessed using a recombinant yeast screen. Environ. Toxicol. Chem. 1996; 15: 241-248.

6. Pelissero C, Menn FL, Kaushick S. Estrogenic effect of dietary soya bean meal on vitellogenesis in cultured Siberian sturgeon Acipenser baeri. Gen. Comp. Endocrinol. 1991; 83:447-457.

7. Pelissero C, Sumpter JP. Steroids and "steroid-like" substances in fish diets. Aquaculture 1992; 107: 283-301. 